\title{
Are US Jurisdictions Prepared to Dispense Medical Countermeasures Through Open Points of Dispensing? Findings from a National Study
}

Terri Rebmann, Travis M. Loux, Zachary Swick, Harlan Dolgin, David Reddick, and Mary Wakefield

Communities will rely on open points of dispensing (PODs) for mass dispensing of medical countermeasures following a bioterrorism attack or a pandemic. US Cities Readiness Initiative (CRI) open POD preparedness is assessed using the Technical Assistance Review (TAR) but focuses on oral prophylaxis dispensing; mass vaccination readiness is not well measured. Non-CRI preparedness had not been studied. In 2013 an online questionnaire was sent to all 456 CRIs and a random sample of 500 non-CRIs to measure open POD preparedness and exercise participation. Hierarchical linear regression was used to describe factors associated with higher POD preparedness and exercise participation scores. In total, 257 subjects participated, for a 41\% response rate. Almost all open PODs have existing written plans and/or a layout for each site $(93.4 \%, n=240$, and $87.0 \%, n=220)$. Only half $(46.7 \%, n=120)$ have an alternative dispensing modality in place, and even fewer $(42.6 \%, n=104)$ report having adequate staffing. Determinants of open POD preparedness were perceived preparedness, participation in more POD exercises, and more closed POD coverage. Most jurisdictions conducted a full-scale exercise and a staff notification drill $(83.7 \%, n=215$ for both). Fewer than half $(40.5 \%, n=104)$ have conducted a vaccination clinic exercise. Determinants of increased POD exercises were perceived preparedness, years of work experience, community type (nontribal), and larger population. Because successful open POD deployment is critical, jurisdictions need to plan for mass vaccination, use of alternative dispensing modalities, and recruitment strategies to increase POD staffing.

$\mathrm{L}$ ARGE-SCALE BIOLOGICAL EVENTS, such as a bioterrorism attack or a pandemic, have the potential to result in significant morbidity and mortality and could have an impact on the security of US residents. In recognition of this threat, the US government in 2004 began funding the Strategic National Stockpile (SNS) as a way to augment state and local public health preparedness efforts. ${ }^{1}$ The SNS is managed by the Centers for Disease Control and Prevention (CDC) to provide critical medical assets, including medical countermeasures (MCMs) such as antibiotics and vaccines, to any location in the US within 12 hours. Although the SNS exists to assist jurisdictions with public

Terri Rebmann, PhD, RN, CIC, is Associate Professor and Director, Institute for Biosecurity; Harlan Dolgin, JD, is an adjunct instructor, Environmental and Occupational Health, Institute for Biosecurity; and Mary Wakefield is a graduate student; all in the Department of Environmental and Occupational Health, Saint Louis University, College for Public Health and Social Justice, Saint Louis, Missouri. Travis M. Loux, PhD, is Assistant Professor, Department of Biostatistics, Saint Louis University, College for Public Health and Social Justice. Zachary Swick, MS, is Domestic Preparedness Planner, Oregon Military Department, Oregon Office of Emergency Management, Portland, OR. David Reddick and Dr. Dolgin are co-owners of Bio-Defense Network, St. Louis, Missouri. 
health preparedness, these federal assets are considered supplemental and are not intended to replace local public health preparedness capabilities. Each jurisdiction is responsible for mass dispensing of MCMs in the event of a pandemic or bioterrorism attack, with the goal of being capable of delivering mass prophylaxis to the entire community within 48 hours. $^{2,3}$ In order to facilitate dispensing of medication in large communities, the CDC created the Cities Readiness Initiative (CRI), a program designed to enhance preparedness in the nation's largest communities. ${ }^{4}$ Starting with 21 cities in 2004, the program has expanded and currently includes 72 Metropolitan Statistical Areas (MSAs), with at least 1 in each state. A CRI jurisdiction is one that receives federal funding to enhance public health preparedness.

Various dispensing modalities have been explored over the past decade to assure MCMs are quickly made available to exposed populations. Potential dispensing modalities may include home delivery of MCMs through the United States Postal Service, ${ }^{1}$ preevent placement of MCMs to central venues in communities, ${ }^{1}$ predeployment of MCMs to first responders and individual homes in the form of MedKits, ${ }^{5}$ and the use of points of dispensing (PODs). Most jurisdictions rely primarily on an extensive network of PODs for mass MCM dispensing. PODs can be either "open" or "closed": All community members are offered access to open POD sites, and closed PODs are available only to the entity hosting the POD. ${ }^{6}$ Although many jurisdictions have begun developing closed PODs, the vast majority of most jurisdictions' population will need to access an open POD to obtain MCMs, because widespread closed POD coverage does not currently exist. ${ }^{6}$

Deploying open PODs is complex and requires extensive planning, dedicated resources, and multiple exercises to assess readiness. Historically, the CDC has used the Technical Assistance Review (TAR) to assess CRI jurisdictions' ability to dispense MCMs (ie, open POD preparedness for deployment). ${ }^{7}$ CDC reports have indicated that not all CRI jurisdictions have received passing grades on the TAR, although the TAR scores have been improving over time. ${ }^{7}$ And there are notable gaps in the TAR's ability to adequately assess jurisdictions for mass dispensing readiness. For example, the TAR is used only to assess CRI jurisdictions, and the TAR focuses strictly on mass dispensing of oral countermeasures and does not address mass vaccination readiness.

Researchers have examined a few aspects of open POD preparedness not measured by the TAR. For example, mathematical modeling has been used to calculate POD throughput $^{8-10}$ and optimal traffic patterns in relation to PODs. ${ }^{11,12}$ POD exercises have been used to assess patient satisfaction, ${ }^{13}$ efficiency of using a head-of-household distribution method, ${ }^{2}$ and patient understanding of POD signage and medication instructions. ${ }^{14}$ Researchers have also used deployment during real events to assess POD preparedness, ${ }^{15-18}$ including the use of PODs for mass dispensing during smaller outbreaks ${ }^{18}$ and cost-effective approaches to mass dispensing. ${ }^{17}$ Despite this research and the TAR assessments, little is known about many aspects of CRI POD preparedness beyond the TAR standards or POD preparedness among non-CRI jurisdictions that are not assessed by the TAR. The purpose of this study is to assess open POD preparedness for mass vaccination, including smallpox vaccine administration, perceived source(s) for jurisdictional POD preparedness priorities, and open POD preparedness and exercise participation across CRI and non-CRI jurisdictions.

\section{Methods}

Public health disaster planners in the US, recruited from all 456 CRIs and a random sample of 500 non-CRIs, were sent the link to an anonymous online survey during a 5-month window in the summer and early fall of 2013. Jurisdictions were contacted by phone, and the recruiter asked to speak with the person responsible for planning of medical countermeasure mass dispensing for the jurisdiction. Willing participants were sent a recruitment email containing a survey link administered through Qualtrics ${ }^{\circledR}$ Software version 2013. A follow-up email was sent 2 weeks later to maximize response rates, following a modified Dillman's Total Design Method. ${ }^{19}$ The Saint Louis University Institutional Review Board approved this study.

\section{Instrument}

This study aimed to measure aspects of open POD preparedness that had not been assessed in previous studies. Therefore, a new questionnaire was created for this study, based on CDC's TAR criteria for open POD preparedness, ${ }^{7}$ a literature review summarizing infection prevention and training recommendations for open PODs, ${ }^{20}$ and findings from a mass dispensing workshop published in a report by the Institute of Medicine. ${ }^{1}$ Items measuring perceived preparedness (ie, perceived ability of a jurisdiction to distribute mass prophylaxis to the entire population within 24 or 48 hours, and/or to administer smallpox vaccine to all citizens within 48 hours and/or 6 days) were developed from CDC POD standards $^{21}$ and recommendations outlined in the CDC Smallpox Response Plan and Guidelines. ${ }^{22}$ The questionnaire was pilot tested by a group of 10 public health professionals from various locations in the US who are responsible for open PODs in their community. Pilot testing consisted of assessing the length of time needed to complete the questionnaire, ease of use, and clarity and thoroughness of items and answer options. Feedback from the pilot testing was then used to refine the instrument. The final 40 -item instrument measures:

1. open POD preparedness (8 indicators);

2. existence of an alternative dispensing modality, nonclosed POD (6 items);

3. existence and coverage of a closed POD network (2 items); 
4. perceived preparedness for open PODs to distribute mass antibiotics and/or vaccine within CDC-recommended timeframes (4 items);

5. perceived source(s) for jurisdictional open POD preparedness priorities (funding agencies, risk analysis, exercise outcomes, and/or administrators; 4 items);

6. open POD exercise participation (6 items);

7. having an after-action report for latest exercise ( 1 item);

8. having addressed deficiencies noted in last after-action report (1 item); and

9. scenario(s) used in open POD exercises (8 items).

\section{Data Analysis}

All data analyses were performed using the Statistical Package for the Social Sciences (SPSS) 22.0 and the R statistical program. ${ }^{23}$ Descriptive statistics were computed for each question and used to describe open POD preparedness and participation in POD exercises or drills. An overall POD preparedness score was calculated by assigning 1 point for each of 8 possible preparedness components that jurisdictions reported having in their POD plan. Four of the 8 POD preparedness indicators were scored 1 or 0 points only: (1) addressing vaccine cold storage in plan, (2) having sufficient cold storage, (3) having at least 1 alternative dispensing modality, and (4) perceived sufficient staffing.

The other preparedness indicators could be partially met and were assigned $1,0.5$, or 0 points: (1) having a written plan for all open PODs, (2) open POD locations/sites supported by memoranda of understanding (MOUs), (3) transportation for medical delivery to PODs supported by MOUs, and (4) having a layout of each planned open POD site.

The highest possible score for POD preparedness was 8 (ie, 1 point for each of the 8 preparedness indicators). A POD exercise participation score was calculated from 4 indicators: (1) whether a full-scale exercise had been conducted within the past 5 years (yes/no), (2) whether a staff or volunteer notification exercise had been conducted within the past 2 years (yes/no), (3) whether the jurisdiction wrote an after-action report following the last POD exercise (yes, in progress, or no), and (4) whether deficiencies identified by the last POD exercise had been addressed by the POD plan (yes, in progress, or no). Each item was scored 1 point; the maximum exercise participation score was 4 points. Hierarchical linear regression was used to describe factors associated with higher POD preparedness and exercise participation scores. Variables that were significant on univariate analysis but nonsignificant on multivariate analysis were dropped from the final models; only final models are reported. A critical $p$-value of 0.05 was used for all analyses.

\section{Results}

During recruitment, 632 public health officials were reached and invited to participate; 20 declined. Of the 20 disaster planners who refused to participate, half $(n=10)$ were employed by a CRI jurisdiction. There were no differences between those who refused and those who agreed to participate in terms of population size (except CRI vs non-CRI) or other demographics, and there was no obvious reason for participation refusal, such as proximity to a previous terrorism target area.

A survey link was emailed to the 612 consenting individuals; 301 surveys were returned. Forty-four questionnaires were removed because of excessive missing data, yielding 257 subjects (41\% response rate). More than half of the disaster planners $(65.1 \%, n=157)$ were female, and most $(85 \%, n=218)$ were employed full time. Age groups were represented almost equally, with slightly more being 56 years or older $(30.7 \%, n=74)$ or aged 46 to 55 years $(27.0 \%, n=65)$ compared with those who were younger. Individuals of various education levels participated, but the majority had either a bachelor's $(41.5 \%, n=100)$ or master's $(38.6 \%, n=93)$ degree. The participants ranged in their years of work experience, but about a third $37.3 \%$, $n=90)$ had between 6 and 10 years of experience. About half $(46.0 \%, n=118)$ reported having some type of medical education; of these, half $(50.8 \%, n=60)$ were nurses, $29.7 \%(n=35)$ were emergency medical technicians, a few $(1.7 \%, n=2)$ were physicians, and some $17.8 \%(n=21)$ reported some other type of unspecified medical education. A little over half $(57 \%, n=147)$ were employed in a CRI; very few $(4.3 \%, n=11)$ worked for a tribal jurisdiction. Approximately half $(53.5 \%, n=129)$ reported living in a jurisdiction with a population of 100,000 or fewer people.

\section{Open POD Preparedness}

Open POD preparedness scores ranged from 0 to 8 points, with an average score of 5.8. The frequency with which jurisdictions reported having each component of open POD preparedness is listed in Table 1. Almost all disaster planners reported that their open PODs have written plans for operation and/or a layout for each open POD site $(93.4 \%, n=240$, and $85.6 \%, n=220$, respectively; Table 1). In contrast, fewer than half $(46.7 \%, n=120)$ have an alternative dispensing modality in place, and even fewer $(42.6 \%, n=104)$ believe they have an adequate number of staff to operate their open PODs (Table 1). Determinants of increased open POD preparedness were: (1) perceived ability to distribute antibiotics to $100 \%$ of population within 48 hours; (2) increased POD Exercise Participation score (ie, participation in more POD exercises during the past 2 years); (3) POD preparedness priorities being determined by public health administrators (as opposed to funding agencies, risk analysis, or some other source) and the existence of closed PODs and the extent of closed POD coverage; and (4) a greater percentage of population covered by closed PODs (Table 2). Being a CRI jurisdiction was not a significant predictor of open POD preparedness. 
Table 1. Open Point of Dispensing (POD) Preparedness Indicators

\begin{tabular}{|c|c|}
\hline POD Preparedness Indicator & $\begin{array}{c}\text { Has Specified Component }(\mathrm{N}=257) \\
\%(\mathrm{n})\end{array}$ \\
\hline Presence of a written plan for open PODs & $93.4(240)$ \\
\hline Plan contains layout of each planned open POD site & $85.6(220)$ \\
\hline POD plan addresses cold storage for vaccines & $79.8(205)$ \\
\hline Perceived sufficient cold storage planned & $73.5(189)$ \\
\hline Open POD locations/sites supported by MOUs & $72.4(186)$ \\
\hline Transportation for medical delivery to POD supported by MOU & $71.2(183)$ \\
\hline Presence of at least 1 alternative dispensing modality & $46.7(120)$ \\
\hline Perceived adequate staffing to operate all open PODs & $42.6(104)$ \\
\hline
\end{tabular}

$\mathrm{MOU}=$ memorandum of understanding.

\section{Perceived Priorities for Preparedness}

Disaster planners were asked to report the extent to which they believed their jurisdiction's POD preparedness priorities were determined by 4 different sources (respondents could select more than one): (1) mandates from funding agencies, (2) results from a jurisdictional risk analysis, (3) POD exercise outcomes, and (4) administrative decision or mandate. Approximately three-quarters reported that their POD preparedness priorities are determined by funding agencies and/or exercise outcomes $(75.4 \%, n=184$, and $73.0 \%, n=178$, respectively). A little more than half $(55.7 \%, n=136)$ indicated that their jurisdictional risk analysis is used to set POD preparedness goals, and just under half $(47.1 \%, n=115)$ reported that priorities are determined by jurisdictional administrators.

\section{Plans and Perceptions}

Almost all jurisdictions $(92.2 \%, n=225)$ are planning to use open PODs to distribute vaccines after a biological event, assuming that a vaccine is available. Three-quarters $(75.8 \%, n=185)$ plan to also use a closed POD(s) to distribute vaccine. Few $(7.0 \%, n=18)$ reported that they do not plan to distribute vaccines through either open or closed PODs after a biological event. Most disaster planners
$(82 \%, n=200)$ believed that their jurisdiction could distribute antibiotics to $100 \%$ of their population within 48 hours, and three-quarters $(75 \%, n=183)$ indicated that they could administer smallpox vaccine to $100 \%$ of their jurisdiction's population within 6 days.

Despite this, fewer than half $(42.6 \%, n=104)$ believed they had adequate numbers of staff and/or volunteers to operate all of their jurisdiction's open PODs. From multiple logistic regression, both individual and jurisdictional level predictors of perceived sufficient open POD staffing were identified (Table 3). A single jurisdiction-level variable was associated with increased perceived adequacy of open POD staffing: being a CRI jurisdiction. Beliefs about POD staffing were also related to 1 respondent-level predictor: the respondent's level of formal education, with more highly educated respondents being less likely to believe they had adequate staffing to run their open PODs. Two beliefs regarding the source(s) of POD planning priorities were also related to increased perceived sufficient staffing: Those who perceived their open POD planning priorities were decided by a funding agency and/or by the jurisdictional risk analysis were more likely to believe their open PODs had adequate staffing. Having at least 1 closed POD and/or the percentage of the jurisdiction population covered by closed PODs were not associated with perceived adequate staffing for open PODs.

Table 2. Determinants of Open Point of Dispensing (POD) Preparedness

\begin{tabular}{lccc}
\hline & \multicolumn{2}{c}{ Open POD Preparedness } \\
\cline { 2 - 4 } Factor & $\beta$ & SE & p-Value \\
\hline Perceived mass dispensing capability within 48 hours $^{\mathrm{a}}$ & 0.793 & $\mathbf{0 . 0 0 1}$ \\
Higher POD Exercise Participation score & 0.491 & 0.24 & $\mathbf{0 . 0 0 1}$ \\
POD preparedness priorities from administrators & 0.387 & 0.12 & $\mathbf{0 . 0 3 0}$ \\
Population covered by closed POD(s) & & & $\mathrm{NS}$ \\
$1-10 \%$ & 0.103 & 0.25 & $\mathrm{NS}$ \\
$11-30 \%$ & 0.276 & 0.27 & $\mathrm{NS}$ \\
$31-50 \%$ & 0.459 & 0.46 & $\mathbf{0 . 0 0 3}$ \\
$\geq 51 \%$ & 1.268 & 0.43 & \\
\hline
\end{tabular}

${ }^{a}$ Perceived ability to distribute antibiotics to $100 \%$ of the population within 48 hours.

${ }^{b}$ Participation in more POD exercises during the past 2 years.

Referent group: Population covered by closed POD: $0 \%$

$\mathrm{NS}=$ nonsignificant. 
Table 3. Perceived Adequate Staffing for Open Points of Dispensing (PODs) by Logistic Regression

\begin{tabular}{lccc}
\hline & OR & $95 \%$ CI & p-Value \\
\hline Preparedness priorities from funding agencies & 2.55 & $(1.23,5.25)$ & $\mathbf{0 . 0 1 1}$ \\
Preparedness priorities from risk analysis & 2.20 & $(1.24,3.88)$ & $\mathbf{0 . 0 0 7}$ \\
CRI jurisdiction & 2.50 & $(1.37,4.56)$ & $\mathbf{0 . 0 0 3}$ \\
Education level & & & \\
$\quad$ Associate's degree & 0.23 & $(0.05,0.94)$ & $\mathbf{0 . 0 4 1}$ \\
Bachelor's degree & 0.24 & $(0.07,0.85)$ & $\mathbf{0 . 0 2 6}$ \\
Master's degree & 0.18 & $(0.05,0.64)$ & $\mathbf{0 . 0 0 8}$ \\
MD/PhD/equivalent & 0.08 & $(0.01,1.29)$ & $\mathrm{NS}$ \\
\hline
\end{tabular}

Referent group: education level: high school, GED, or some college.

$\mathrm{OR}=$ odds ratio; $\mathrm{CI}=$ confidence interval; $\mathrm{NS}=$ nonsignificant .

\section{Alternative Dispensing Modalities}

Jurisdictions were asked if they currently have various types of alternative dispensing modalities in place, including a drive-through POD, a plan to use the USPS to deliver medication, or preplacement of MedKits. Fewer than half $(46.7 \%, n=120)$ have any type of alternative dispensing modality currently in place (Table 1). Fewer than half $(44.1 \%, n=113)$ plan to deploy a drive-through POD, though an additional $33.6 \%(n=86)$ are considering using one but did not yet have a plan in place. Of those who have a drive-through POD plan $(n=113)$, three-quarters $(73.2 \%$, $n=82)$ plan to distribute both oral medical countermeasures and vaccine through the drive-through; $21.4 \%(n=24)$ intend to dispense only oral countermeasures.

Very few jurisdictions currently have a plan in place to distribute MCMs via the USPS $(4.7 \%, n=12)$, though almost a third $(38.4 \%, n=98)$ stated that they are considering it. The least frequently reported alternative dispensing modality was the use of preplaced MCMs in the form of antibiotics (ie, MedKits). Very few jurisdictions $(1.2 \%, n=3)$ have MedKits in place, and only $9.4 \%$ $(n=24)$ are considering them in the future. When asked to identify the primary reason they are not currently using MedKits, a third $(33.1 \%, n=78)$ indicated that they are too expensive, $26.3 \%(n=62)$ reported having never heard of this option, $16.9 \%(n=40)$ cited a lack of standards or protocols for their use, and $16.1 \%(n=38)$ reported that they are not available in their jurisdiction.

The existence of closed PODs and the extent of closed POD coverage for these jurisdictions were also assessed in this study; the results have been published previously. ${ }^{6}$ Three-quarters of jurisdictions $(74.2 \%, n=181)$ have at least 1 closed POD. Almost all jurisdictions with a closed POD(s) have $1 \%$ to $10 \%$ or $11 \%$ to $30 \%$ of their population covered by a closed $\mathrm{POD}(\mathrm{s})\left(47 \%\right.$ and $38.7 \%$, respectively). ${ }^{6}$

\section{Exercise Participation and Scenarios Used}

Open POD exercise participation was measured in 2 ways: (1) extent to which PODs met exercise standards (POD
Exercise Standards score; conducted a full-scale exercise in the past 5 years, performed a staff and volunteer notification drill in the past 2 years, wrote an after-action report after the last exercise, and addressed the gaps identified in the after-action report in the POD plan), and (2) extent to which open PODs conducted a variety of exercises in the past 2 years (POD Exercise Participation score; 6 types of exercises assessed). The POD Exercise Standards score ranged from 0 to 4 points, with an average score of 3.1 . Most jurisdictions reported that a full-scale exercise had been conducted within the past 5 years and that a staff and volunteer notification drill had been performed in the past 2 years (83.7\%, $n=215$ for both). Almost all (84.4\%, $n=217$ ) indicated that an after-action report had been written after the most recent exercise. However, fewer than a third $(26.8 \%, n=69)$ reported that the deficiencies identified during the last POD exercise have been addressed in the POD plan. From linear regression, determinants of increased POD Exercise Standards scores were as follows: (1) perceived ability to distribute antibiotics to $100 \%$ of the population within 48 hours, (2) being a CRI jurisdiction, and (3) a greater percentage of population covered by a closed POD (Table 4).

The POD Exercise Participation score related to how many exercises had been conducted in the past 2 years ranged from 0 to 6 points, with an average score of 3.5. As mentioned previously, most jurisdictions $(83.7 \%, n=215)$ had conducted a staff and volunteer notification drill in the past 2 years. The next most frequently reported exercises in the past 2 years included a facility set-up drill $(68.0 \%$, $n=166)$ or a POD throughput assessment $(63.5 \%$, $n=155)$. About half had conducted either a pick list generation drill $(54.1 \%, n=132)$ or a full-scale exercise $(52.5 \%, n=128)$ in the past 2 years. Fewer than half $(47.1 \%, n=115)$ had actually deployed their POD in the past 2 years and evaluated its performance. From linear regression, determinants of increased POD Exercise Participation scores in the past 2 years were as follows: (1) perceived ability to distribute antibiotics to $100 \%$ of the population within 24 hours, (2) perceived ability to distribute antibiotics to $100 \%$ of the population within 48 hours, (3) respondent's years of work experience, (4) being 
Table 4. Determinants of Open Point of Dispensing (POD) Exercise Participation

\begin{tabular}{|c|c|c|c|c|c|c|}
\hline \multirow[b]{2}{*}{ Factor } & \multicolumn{3}{|c|}{ Exercise Standards } & \multicolumn{3}{|c|}{ Exercise Participation } \\
\hline & $\beta$ & $S E$ & $\mathrm{p}$-Value & $\beta$ & $S E$ & $\mathrm{p}$-value \\
\hline Perceived mass dispensing capability within 24 hours $^{\mathrm{a}}$ & & & & 0.716 & 0.26 & 0.006 \\
\hline Perceived mass dispensing capability within 48 hours $^{\mathrm{a}}$ & 0.388 & 0.12 & 0.002 & 0.743 & 0.32 & 0.020 \\
\hline CRI jurisdiction & 0.214 & 0.10 & 0.033 & & & \\
\hline \multicolumn{7}{|l|}{ Population covered by closed POD(s) } \\
\hline $1-10 \%$ & 0.371 & 0.13 & 0.004 & & & \\
\hline $11-30 \%$ & 0.627 & 0.14 & $<0.001$ & & & \\
\hline $31-50 \%$ & 0.625 & 0.24 & 0.011 & & & \\
\hline$\geq 51 \%$ & 0.496 & 0.22 & 0.026 & & & \\
\hline \multicolumn{7}{|l|}{ Years of work experience } \\
\hline $2-5$ years & & & & 1.096 & 0.69 & NS \\
\hline $6-10$ years & & & & 1.549 & 0.61 & 0.012 \\
\hline$\geq 11$ years & & & & 1.025 & 0.61 & NS \\
\hline Jurisdiction a tribal community & & & & -1.311 & 0.55 & 0.019 \\
\hline \multicolumn{7}{|l|}{ Jurisdiction population } \\
\hline $100,001-500,000$ & & & & 0.417 & 0.26 & NS \\
\hline $500,001-999,999$ & & & & 1.819 & 0.37 & $<0.001$ \\
\hline$\geq 1$ million & & & & 1.469 & 0.46 & 0.002 \\
\hline
\end{tabular}

${ }^{\text {a }}$ Perceived ability to distribute antibiotics to $100 \%$ of the population within 24 or 48 hours.

Referent groups: Population covered by closed POD: 0\%; Years of employment experience: $\leq 1$ year; Jurisdiction population: $\leq 100,000$

$\mathrm{NS}=$ nonsignificant

a nontribal community, and (5) greater jurisdiction population (Table 4). Being a CRI jurisdiction was not a significant predictor of POD exercise participation.

Disaster planners were asked to identify which infectious disease scenario(s) had been used in an open POD exercise within the past 2 years; results are listed in Table 5. The most frequently reported scenarios included anthrax $(50.2 \%, n=129)$ and vaccination clinics $(40.5 \%, n=104$; Table 5). The least frequently reported infectious disease exercise scenarios used in the past 2 years included botulism $(1.6 \%, n=4)$ and viral hemorrhagic fever $(0.4 \%, n=1$; Table 5).

\section{Discussion}

This study found that many jurisdictions are taking important steps to become prepared to dispense medical countermeasures on a mass scale through open PODs. Examples include having a written plan for open PODs and having a layout of each open POD in the plan. However, multiple critical aspects of POD preparedness are not being addressed by all jurisdictions, and $20 \%$ of the disaster planners in this study reported that they do not believe their jurisdiction could meet the CDC standard of mass dispensing MCMs to all citizens in 48 hours. Gaps in planning across multiple jurisdictions include a failure to address cold storage for vaccines, not having sufficient cold storage available on-site at the POD, and not having written memoranda of agreement for POD sites and medication transportation.

One of the most significant gaps in open POD preparedness identified in this study is that although almost all of the participating disaster planners reported that they believe their jurisdiction can distribute antibiotics to their entire population in 48 hours, fewer than half reported that

Table 5. Infectious Disease Scenarios Used in Open Point-of-Dispensing (POD)

Exercises in the Past 2 Years

\begin{tabular}{lc}
\hline Infectious Disease Scenario & $\begin{array}{c}\text { Used in the Past } 2 \text { Years }(\mathrm{N}=257) \\
\%(\mathrm{n})\end{array}$ \\
\hline Anthrax & $50.2(129)$ \\
Vaccination clinic & $40.5(104)$ \\
Pandemic influenza & $39.7(102)$ \\
Pneumonic plague & $7.8(20)$ \\
Tularemia & $3.5(9)$ \\
Smallpox & $2.7(7)$ \\
Botulism & $1.6(4)$ \\
Viral hemorrhagic fever (eg, Ebola, Marburg) & $0.4(1)$ \\
\hline
\end{tabular}


they have adequate staff and/or volunteers to operate their open PODs. It is unclear why so many jurisdictions reported perceived readiness to deploy despite not having adequate workers for the POD. Perhaps this reflects wishful thinking about the current state of preparedness for biological events. This attitude was encountered during the Ebola outbreak in fall 2014, when US hospitals initially reported being fully prepared to care for Ebola patients safely, but gaps in readiness became apparent when occupationally acquired transmission occurred. ${ }^{24}$ Wishful thinking about biological preparedness can result in mistakes and increased morbidity and mortality. Without adequate staffing, it is highly unlikely that open PODs could successfully meet their goals. Millions of dollars and countless hours have been invested in US public health preparedness, yet this study's findings indicate that there are still large gaps in readiness to respond to a biological event. Furthermore, large-scale biological events continue to occur regularly; Ebola and Middle East respiratory syndrome coronavirus (MERS Co-V) are just 2 recent examples. More robust mass medical countermeasure planning is needed to ensure the health security of US citizens.

In this study, one predictor of higher POD preparedness was having more of the jurisdiction covered by closed $\operatorname{POD}(\mathrm{s})$; this finding makes sense, given that researchers have estimated that closed PODs can reduce the burden from open PODs by $40 \%$ to $50 \% .{ }^{1}$ Despite that, this study found that having at least 1 closed POD and/or the percentage of the jurisdiction population covered by closed PODs were not associated with perceived adequate staffing for open PODs.

These findings are significant because they indicate that having closed POD coverage does not alleviate the challenge of having sufficient staffing for open PODs. Even jurisdictions that reported having extensive closed POD coverage in their community still reported not having adequate staffing to deploy their open PODs. Recruitment of volunteers should remain a high priority for open POD planning to better ensure successful deployment. ${ }^{25}$ Too few POD workers could result in decreased throughput, chaotic conditions at the POD, and/or "walkaways," leaving community members at risk from the biological event. ${ }^{26}$

One option for POD recruitment efforts is to focus on nearby healthcare professional education programs, such as nursing and medical schools, as a means of obtaining volunteers for the POD. These students could easily be trained for various roles in an open POD, including dispensing medication and administering vaccine. Involving future medical professionals in POD planning also raises their awareness of biological event preparedness and response, an issue of vital importance for all healthcare professionals.

An option to minimize the number of needed POD workers is to decrease the burden on open POD throughput by implementing alternative dispensing modalities. 1,5 The CDC TAR indicates that jurisdictions should use at least 1 alternative dispensing modality in addition to open
PODs for mass dispensing of medical countermeasures. ${ }^{7}$ However, this study found that fewer than half of all jurisdictions have at least 1 alternative dispensing modality in place. Of those that had an alternative dispensing modality, drive-through PODs were the most frequent type in place. Researchers indicate that drive-through PODs represent a feasible and effective method of mass MCM dispensing by increasing access to MCMs, especially among minority and underinsured individuals. ${ }^{27,28}$ An SNS dispensing exercise conducted in Hawaii using drive-through PODs found that, although special services such as those for the sight or hearing impaired were limited in this model, it was highly accurate and time efficient overall. ${ }^{27}$ The researchers noted that the drive-through POD was especially useful in rural areas that had limited resources; ${ }^{27}$ therefore, this alternative dispensing modality should be appealing to many non-CRI jurisdictions. Although it was not assessed in this study, the use of existing pharmacies and healthcare systems in the jurisdiction may also be used to distribute MCMs. This would further decrease the burden on open and closed PODs in communities.

Few disaster planners in this study reported that their jurisdictions plan to use USPS MCM delivery or preplacement of MedKits as an alternative dispensing modality. Neither USPS delivery nor use of MedKits may be as feasible as drive-through PODs because of ethical and logistical issues involved in these programs.

In 2009, the president issued an executive order outlining the intent to implement or consider implementing the US Postal Service Model. ${ }^{29}$ The USPS model was conceptualized as a way to rapidly dispense oral MCMs to all US households, while reducing the burden on open PODs, by having mail carriers with security escorts deliver oral MCMs directly to homes. The primary advantage of using the USPS model is that the infrastructure for rapid mass delivery to every household already exists. However, the USPS model is considered controversial by many disaster planners (H. Milam, Disaster Planner, St. Charles County Department of Health, personal communication, 2013). The primary criticism of the USPS model is that it does not allow for proper screening to be sure that the individuals in the household are receiving the correct medications in the appropriate dosage. At PODs, individuals are screened for contraindications to the standard medications, and different MCMs may be substituted when necessary. Using the USPS model makes this screening process much more challenging, which means that it might not be done correctly. Individuals could receive an inappropriate medication, leading to an allergic reaction or suboptimal clinical response. Another major criticism of the USPS model is that it would be very difficult to address language barrier and/or reading literacy needs of citizens, which could result in unsafe dosing. A third major limitation is that the model requires a large investment of resources from local law enforcement agencies, because it is predicated on there being 1 security officer with each postal 
worker while delivering medical countermeasures. ${ }^{30}$ The lack of support for this alternative dispensing modality was demonstrated by the very few jurisdictions that currently plan to implement it, though the USPS model has been discussed since $2004 .^{29}$

Disaster planners in this study also reported very little support for MedKits as an alternative dispensing modality; the vast majority of jurisdictions are not even considering this program. MedKits involve preevent placement in individual homes of caches of antibiotics or other MCMs that could be used during a declared public health emergency. ${ }^{1,31}$ MedKits could potentially sit in households for years without ever being used, but they would need to be refreshed if or when the medications expire. Another possible disadvantage to MedKits is that household members' medical conditions could change over time-for example, as a result of pregnancy or development of a condition that changes their contraindications for medications contained in the MedKit; this could result in the MedKit's being unusable during an actual event. The major advantage to this system is that household members would have the correct MCMs on hand when an event occurs, eliminating the need for mass dispensing postevent.

Like the USPS model, MedKits are controversial among some disaster planners. The primary concern is that individuals would take the medications to treat a minor infection of some kind before an event occurs and then not have access to the MCMs postevent when they are needed. $^{32}$ Critics believe that MedKits are simply too tempting for the general public, and their use could possibly contribute to antimicrobial resistance in the community if individuals misused them. ${ }^{32}$

A pilot study using approximately 4,200 MedKits was conducted in St. Louis, MO, in 2006. Households were provided the MedKit and then evaluated at the end of the study period to determine if the kit was still intact or if it had been opened. There were 174 households lost to follow-up, but data are complete for 4,076 households, equaling 12,040 people. In the study, $97 \%$ of all study respondents returned the MedKits fully intact at the end of the study. ${ }^{31}$ Of those who opened the MedKit, almost everyone reported that they did so without removing anything and only opened it because they were curious about what was inside. ${ }^{31}$ Only 4 households opened and used their MedKits: An elderly woman used it during a declared emergency for a storm, 2 households gave it to family members who had sore throats, and 1 household refused to state why the MCMs were taken. ${ }^{31}$ When the study ended, $94 \%$ of the participants reported that they would like to have a MedKit in their home all the time. The study concluded that the use of MedKits was quite successful in that very few households misused the MCMs and almost all study participants reported that they would be willing to purchase MedKits to keep in their homes. ${ }^{31}$

Despite this, the MedKit prototype has not yet received approval from the US Food and Drug Administration
(FDA), though it has been under discussion since 2007. Further studies examining the feasibility of MedKits are currently under way. Given the high percentage of jurisdictions in this current study that reported a lack of adequate staffing for their open PODs, it seems prudent for disaster planners to more actively pursue alternative dispensing modalities, such as drive-through PODs, USPS delivery, and/or preplacement of MedKits. Alternative dispensing modalities decrease the burden on open PODs and increase their likelihood of successful deployment.

Another important finding from this study is the lack of preparedness for mass vaccination at many jurisdictions. Though almost all jurisdictions reported that they are planning for mass vaccination using open and/or closed PODs, a small percentage of jurisdictions (7\%) reported that they do not plan to distribute vaccines through either open or closed PODs after a biological event. These findings are curious, as it makes one wonder what these jurisdictions plan to do during biological events requiring mass vaccination. Will mass vaccination not occur in those communities? Perhaps those public health departments have a plan to use neighboring jurisdictions' mass vaccination processes to protect their citizens. More research needs to be conducted in this area to determine the reasons for failure to plan for mass vaccination, as it leaves those populations at high risk of morbidity and mortality during biological events requiring mass vaccination.

Though almost all jurisdictions are planning to use open PODs to distribute vaccines after a biological event, a large number of jurisdictions are not conducting exercises to assess their ability to mass vaccinate citizens. More than 90\% of jurisdictions reported that their open PODs will dispense vaccine when needed, but only $40 \%$ have conducted a vaccine clinic exercise during the past 2 years and very few (fewer than 3\%) have used a smallpox scenario in a POD exercise. Furthermore, only three-quarters of participating jurisdictions reported that their POD could successfully mass vaccinate their citizens against smallpox within 6 days. According to the CDC, smallpox vaccine is most effective when given within 3 days of exposure, but it will provide limited protection if administered within 4 to 7 days postexposure. $^{22}$ Therefore, it is prudent to plan for smallpox mass vaccination to be completed in fewer than 7 days. POD exercises are vital to ensure that mass dispensing of MCMs can be accomplished within expected timelines. ${ }^{16}$

An interesting finding from this study is that jurisdictions participating in more POD exercises had higher POD preparedness scores compared to jurisdictions conducting fewer exercises. This provides evidence that POD exercises are associated with higher confidence of successful POD deployment. Disaster planners from less populated areas and tribal jurisdictions reported conducting significantly fewer POD exercises compared to more populated regions and nontribal jurisdictions. Regulatory compliance and funding are likely 2 reasons for this finding: CRI jurisdictions have been evaluated based on the TAR criteria, which include exercise standards, 
and they have funding contracts from federal and state agencies that include exercise requirements $(\mathrm{H}$. Milam, personal communication, 2013). Non-CRI jurisdictions (ie, less populated regions) are not evaluated on TAR criteria and may not necessarily have funding-based contracts, which reduce the incentive to conduct resource-intensive exercises (H. Milam, personal communication, 2013). It is vital that all jurisdictions regularly conduct exercises to assess POD readiness to deploy; written plans are only the first step. ${ }^{33}$

Performing a full-scale activation of an open POD every few years is not an adequate means of measuring or assuring preparedness. Staff and volunteer turnover, changes in procedure, and ever-evolving infectious disease threats make disaster planning an ongoing challenge requiring frequent assessments to determine readiness. In addition, it is critical that scenarios involving both mass dispensing of oral countermeasures and mass vaccination be used in POD exercises, so that all aspects of POD preparedness can be assessed. POD exercises can consist of simulated disasters or actual deployment during an event. For example, Rhode Island tested its mass vaccination plan during the 2009 H1N1 influenza pandemic using multiple PODs to deliver the $\mathrm{H} 1 \mathrm{~N} 1$ vaccine. ${ }^{33}$

The major strengths of this study are that it is the first nationwide study to examine POD preparedness for both CRI and non-CRI jurisdictions, and it is one of the first to assess aspects of POD preparedness not covered by TAR criteria, such as jurisdictions' ability to mass vaccinate. As previously discussed, the TAR criteria were limited and did not adequately address many critical aspects of public health preparedness, such as readiness for mass vaccination. It should be noted that in fall 2014, the CDC began pilot testing a new assessment tool for public health preparedness, called the Operational Readiness Review Tool (ORR), with the intent of rolling it out nationwide in $2015 .{ }^{34}$ The ORR did not exist when this study was conducted, and findings from the ORR pilot are not yet available and thus could not be used for comparison to this study's findings. Implementation of the ORR demonstrates the CDC's recognition of the limitations of the TAR and is an important step toward more thorough assessments of jurisdictional public health preparedness.

One limitation of this study is that there were significantly more CRI respondents compared to non-CRI participants, though both groups were approached equally for recruitment. This increases the risk of bias slightly, but, given that there were few reported differences between CRI versus nonCRI jurisdictions, this risk is minimized. In addition, the randomized sampling method for non-CRI recruitment and the nationwide sample help increase generalizability.

\section{Conclusions}

Findings from this study illustrate open POD planning and exercise gaps in both CRI and non-CRI jurisdictions that are not currently being monitored by the CDC TAR or other performance measures. Examples include a lack of planning and exercises for mass vaccination, insufficient staffing for open PODs in almost half of all jurisdictions, and no planned use of an alternative dispensing modality. These gaps could result in unsuccessful POD deployment if they are not addressed preevent, leaving community members at risk of increased morbidity and mortality. Jurisdictions need to focus on staff recruitment, planning for mass dispensing of both oral and injectable medical countermeasures, and exercises that assess POD deployment readiness. In addition, it is incumbent on public health leaders to continue exploring alternative dispensing modalities, as they decrease the burden on open PODs and increase community resilience.

\section{REFERENCES}

1. Davis M, Kammersell MS, Altevogt BM. Dispensing Medical Countermeasures for Public Health Emergencies: Workshop Summary. Washington, DC: Institute of Medicine; 2008.

2. Agocs M, Fitzgerald S, Alles $S$, et al. Field testing a head-ofhousehold method to dispense antibiotics. Biosecur Bioterror 2007;5(3):255-267.

3. Hupert N, Xiong W, King K, et al. Uncertainty and operational considerations in mass prophylaxis workforce planning. Disaster Med Public Health Prep 2009;3(Suppl 2): S121-S131.

4. Cities Readiness Initiative. Centers for Disease Control and Prevention website. 2010. http://emergency.cdc.gov/cri/. Accessed August 4, 2013.

5. Nelson CD, Willis HH, Chan EW, Shelton SR, Parker AM. Federal initiative increases community preparedness for public health emergencies. Health Aff (Millwood) 2010; 29(12):2286-2293.

6. Rebmann T, Loux TM, Swick Z, et al. A national study examining closed points of dispensing (PODs): existence, preparedness, exercise participation, and training provided. Biosecur Bioterror 2014;12(4):208-216.

7. Centers for Disease Control and Prevention. Appendix 2: Cities Readiness Initiative technical assistance review scores by metropolitan statistical area and individual planning jurisdictions. In: Public Health Preparedness: 2011 State-byState Update on Laboratory Capabilities and Response Readiness Planning. Centers for Disease Control and Prevention, Office of Public Health Preparedness and Response; September 2011. http://www.cdc.gov/phpr/pubs-links/2011/ documents/SEPT_UPDATE_REPORT_9-13-2011-Final.pdf. Accessed February 4, 2015.

8. Pietz F, Benecke B, Lee EK, Hung Chen C. Modeling and optimizing the public-health infrastructure for emergency response. Interfaces 2009;39(5):476-490.

9. Whitworth MH. Designing the response to an anthrax attack. Interfaces 2006;36(6):562-568.

10. Aaby K, Abbey RL, Herrmann JW, Treadwell M, Jordan CS, Wood K. Embracing computer modeling to address pandemic influenza in the 21st century. J Public Health Manag Pract 2006;12(4):365-372. 
11. Baccam P, Willauer D, Krometis J, Ma Y, Sen A, Boechler M. Mass prophylaxis dispensing concerns: traffic and public access to PODs. Biosecur Bioterror 2011;9(2):139-151.

12. Ma Y, Willauer D, Krometis J, Sen A, Baccam S. Site considerations for points of dispensing after biological terrorist attack: integrated role of transportation planning and medical services. Transport Res Rec 2011;2234:51-61.

13. Ablah E, Scanlon E, Konda K, Tinius A, Gebbie KM. A large-scale points-of-dispensing exercise for first responders and first receivers in Nassau County, New York. Biosecur Bioterror 2010;8(1):25-35.

14. Stergachis A, Wetmore CM, Pennylegion M, et al. Evaluation of a mass dispensing exercise in a Cities Readiness Initiative setting. Am J Health Syst Pharm 2007;64(3):285-293.

15. Saha $S$, Dean B, Teutsch $S$, et al. Efficiency of points of dispensing for influenza $\mathrm{A}(\mathrm{H} 1 \mathrm{~N} 1) \mathrm{pdm} 09$ vaccination, Los Angeles County, California, USA, 2009. Emerg Infect Dis 2014;20(4):590-595.

16. Rinchiuso-Hasselmann A, McKay RL, Williams CA, et al. Protecting the public from H1N1 through points of dispensing (PODs). Biosecur Bioterror 2011;9(1):13-21.

17. Kansagra SM, McGinty MD, Morgenthau BM, et al. Cost comparison of 2 mass vaccination campaigns against influenza A H1N1 in New York City. Am J Public Health 2012; 102(7):1378-1383.

18. Ngo VP, Civen RH, Dassey DE, Davenport D, Mascola L. Using a point-of-dispensing clinic for prophylaxis of meningococcal disease. Biosecur Bioterror 2010;8(1):45-51.

19. Dillman D, Smyth JD, Christian LM. Internet, Mail, and Mixed-Mode Surveys: The Tailored Design Method. 3rd ed. Hoboken, NJ: Wiley; 2008.

20. Rebmann T, Coll B; 2009 APIC Emergency Preparedness Committee. Infection prevention in points of dispensing. Am J Infect Control 2009;37(9):695-702.

21. Centers for Disease Control and Prevention. Point of Dispensing (POD) Standards. 2008. http://health.mo.gov/emergencies/ sns/pdf/12-PODStandards.pdf. Accessed August 4, 2013.

22. Smallpox response plan and guidelines (version 3.0). Centers for Disease Control and Prevention website. Updated June 23, 2004. http://www.bt.cdc.gov/agent/smallpox/responseplan/. Accessed August 4, 2013.

23. R. Core Team. R: A Language and Environment for Statistical Computing. In: R Foundation for Statistical Computing. Vienna, Austria. 2012. http://www.r-project.org. Accessed August 4, 2013.

24. Copeland L. Are U.S. hospitals prepared for possible Ebola battle? USA Today October 14, 2014. http://www.usatoday. com/story/news/nation/2014/10/14/us-hospitals-preparefor-ebola/17221077/. Accessed February 4, 2015.

25. Aakko E, Weed N, Konrad R, Wiesman J. Rethinking volunteer management using a centralized volunteer staging and training area. Disaster Med Public Health Prep 2008;2(2):127-129.

26. Spitzer JD, Hupert N, Duckart J, Xiong W. Operational evaluation of high-throughput community-based mass prophylaxis using just-in-time training. Public Health Rep 2007;122(5): 584-591.

27. Zerwekh T, McKnight J, Hupert N, Wattson D, Hendrickson L, Lane D. Mass medication modeling in response to public health emergencies: outcomes of a drive-thru exercise. J Public Health Manag Pract 2007;13(1):7-15.

28. Carrico R, McKinney WP, Watson NA, Wiemken T, Myers J. Drive-thru influenza immunization: fifteen years of experience. J Emerg Manag 2012;10(3):228-232.

29. National Postal Model for the Delivery of Medical Countermeasures. Departments of Health and Human Services, Homeland Security, Defense, and Justice and the United States Postal Service. 2009. www.phe.gov/Preparedness/ planning/postal/Pages/default.aspx. Accessed February 4, 2015.

30. Stroud C, Nadig L, Altevogt BM. Medical Countermeasures Dispensing: Emergency Use Authorization and the Postal Model. Washington, DC: National Academies Press; 2010.

31. Centers for Disease Control and Prevention. CDC's Division of Strategic National Stockpile Emergency MedKit Evaluation Study Summary. November 15, 2007. http://www. bt.cdc.gov/cri/pdf/medkit-evaluation-summary-2007updated. pdf. Accessed February 4, 2015.

32. Reilly C, and the American Society of Health-System Pharmacists. Best Practices for Distribution of Antivirals for Pandemic Influenza: Public Health Impact and Research Recommendations. Oral testimony provided at the U.S. Food and Drug Administation Joint Meeting of the Antiviral Drugs Advisory Committee and the Nonprescription Drugs Advisory Committee. October 29, 2008. http://www.fda.gov/ ohrms/dockets/ac/08/slides/2008-4385s1-14-AssociationsASHP.pdf. Accessed March 4, 2015.

33. Balbi DA, Calcagni K. Effects of the design and practice of points-of-dispensing on patient-reported satisfaction: municipal H1N1 clinics in Rhode Island. Disaster Med Public Health Prep 2011;5(2):106-111.

34. New resources to assist with operational readiness review tool completion. National Association of County and City Health Officials website. 2014. http://nacchopreparedness. org/?p=3069. Accessed February 4, 2015.

Manuscript received November 6, 2014;

accepted for publication December 15, 2014.

Address correspondence to: Terri Rebmann, PhD, RN, CIC Associate Professor Director, Institute for Biosecurity Saint Louis University, College for Public Health \& Social Justice 3545 Lafayette Room 463 St Louis, MO 63104

E-mail: rebmannt@slu.edu 